ORIGINAL ARTICLE

\title{
Interferential Current Stimulation for Swallowing Disorders in Chronic Obstructive Pulmonary Disease: A Preliminary Study
}

\author{
Yuki Yoshimatsu, MD, PhD ${ }^{\text {a,b }}$ Kazunori Tobino, MD, PhD ${ }^{\text {a,c }}$ Saori Nishizawa, MD a Kohei Yoshimine, MD ${ }^{\text {a }}$ \\ and Yoshitaka Oku MD, PhD ${ }^{b}$
}

\begin{abstract}
Objectives: Swallowing function is affected in patients with chronic obstructive pulmonary disease (COPD), putting them at risk of exacerbation of COPD. We previously reported the effectiveness of the repetitive saliva swallowing test (RSST) in screening for patients at risk of COPD exacerbation. However, evidence on how to improve swallowing function in this population is extremely limited. Interferential current transcutaneous electrical sensory stimulation (IFCTESS) stimulates the larynx and pharynx, thereby improving their sensory function. IFC-TESS is an emerging tool to enhance airway protection and increase swallowing frequency; however, its safety and efficacy in patients with COPD is unknown. Therefore, we performed a preliminary prospective study focusing on stable COPD patients. Methods: Patients with stable COPD who were hospitalized for yearly evaluation were recruited. Patients were included if their RSST was 5 or less. Nurses carried out IFC-TESS twice daily for 10 days. Swallow screening results were compared before and after the 10-day intervention. Results: Ten patients were included in the study. The IFC-TESS intervention was performed safely. Patients and nurses reported no discomfort or concerns regarding the intervention. The EAT-10 and RSST scores improved significantly after the intervention, and tongue pressure also tended to improve. Conclusions: IFC-TESS may be a promising intervention to improve swallowing in patients with COPD who are easily fatigued and struggle to perform swallowing exercises.
\end{abstract}

Key Words: aspiration; COPD; dysphagia; exacerbation; rehabilitation

\section{INTRODUCTION}

Swallowing disorders in chronic obstructive pulmonary disease (COPD) have become a major topic of concern in recent years. It is known that breathing-swallowing coordination is affected in COPD patients and, in addition to the effects of tobacco smoking, lung hyperinflation, aging, sarcopenia, gastroesophageal reflux disease, and dry mouth, breathing-swallowing coordination is likely an important factor related to swallowing disorders in these patients. ${ }^{1,2}$ Dysphagia is increasingly recognized as a critical phenotype in COPD that is prone to exacerbation. ${ }^{3)}$ In our previous study, we investigated the efficacy of the repetitive saliva swallowing test (RSST) in screening for COPD patients who are at risk of exacerbation. ${ }^{4,5)}$ The RSST, in which patients are asked to swallow as many times as possible in $30 \mathrm{~s}$, is a simple and noninvasive method used to screen for dysphagia. Patients with an RSST score of 5 or less were found to be at risk of COPD exacerbation. ${ }^{5)}$

The mechanisms and appropriate screening methods for COPD exacerbation are being clarified, and the next step is to investigate effective ways to treat this population; however,

Received: September 24, 2021, Accepted: January 24, 2022, Published online: February 17, 2022

${ }^{a}$ Department of Respiratory Medicine, Iizuka Hospital, Iizuka, Japan

${ }^{\mathrm{b}}$ Department of Physiology, Hyogo College of Medicine, Nishinomiya, Japan

${ }^{c}$ Department of Respiratory Medicine, Juntendo University Graduate School of Medicine, Tokyo, Japan

Correspondence: Yuki Yoshimatsu, MD, PhD, 3-83 Yoshiomachi, Iizuka, Fukuoka, Japan 820-8505, E-mail: yukitsukihana0105@ gmail.com

Copyright (C) 2022 The Japanese Association of Rehabilitation Medicine

(c) (1) $(-)$ This is an open-access article distributed under the terms of the Creative Commons Attribution Non-Commercial No Derivatives (CC BY-NC-ND) 4.0 License. http://creativecommons.org/licenses/by-nc-nd/4.0/ 
evidence on interventions to improve swallowing function is still extremely limited. It is hoped that physiotherapy and swallow training may be effective. However, the fatigability of patients with COPD makes it difficult for them to perform exercises; moreover, the availability of speech therapists is often limited. Therefore, intervention that does not require physical effort by the patient or the involvement of a speech therapist is desired.

Two electrical stimulation methods have been developed in dysphagia rehabilitation: neuromuscular electrical stimulation (NMES) and transcutaneous electrical sensory stimulation (TESS). NMES evokes muscle contractions to improve motor function and has proven to be effective in a variety of causative conditions. NMES is already widely utilized in clinical practice. In contrast, TESS stimulates the peripheral sensory nerves in the larynx and the pharynx to improve sensory function. Interferential current (IFC)-TESS is emerging as a way to enhance airway protection and increase swallowing frequency.

In basic animal research with guinea pigs, IFC-TESS shortened the swallow delay time, suggesting that it could enhance the pharyngolaryngeal sensory afferent pathway, subserving excitatory inputs to the swallowing pattern generator, and hence facilitate the swallowing reflex. ${ }^{6}$ In clinical studies, IFC-TESS was reportedly effective in dysphagic patients with dementia, ${ }^{7)}$ aspiration pneumonia, and other conditions. ${ }^{8)}$ However, its efficacy in COPD patients is unknown. As a noninvasive method of swallow training that does not require the presence of a speech therapist and does not require any patient effort, IFC-TESS may be a suitable method for swallow training in patients with COPD. Consequently, we performed an exploratory study to investigate the safety and feasibility of IFC-TESS in patients with COPD. To the best of our knowledge, this is the first study in which IFC-TESS was investigated in patients with COPD.

\section{MATERIALS AND METHODS}

\section{Patients}

This was a single-center, prospective, cohort study performed at Iizuka Hospital Department of Respiratory Medicine. Patients with stable COPD who were hospitalized for yearly evaluation and rehabilitation between August 1, 2020, and February 28, 2021, were recruited. COPD was diagnosed according to the GOLD 2017 criteria. ${ }^{9)}$ The inclusion criteria were patients with an RSST of five or less per $30 \mathrm{~s}$ because this group is at high risk of COPD exacerbation. ${ }^{4}$ Patients with a pacemaker or implantable cardioverter defibrillator were excluded because IFC-TESS is contraindicated in such patients. Patients with apparent swallowing disorder caused by other conditions were also excluded. Ethical approval was provided by the Iizuka Hospital Ethics Committee in accordance with the Declaration of Helsinki (Number 20088), and written informed consent was obtained from all patients.

\section{Methods}

The study design is shown in Fig. 1. Demographic information and lung function data were extracted from medical records. Any history of moderate to severe COPD exacerbation in the previous year was assessed from medical records and patient recollection. COPD exacerbation was defined as the presence of new or worsening COPD symptoms (cough, sputum, wheezing, dyspnea, or chest tightness), at least one of which lasted for 3 days or longer.

Upon inclusion, patients underwent swallow screening with the 10-item Eating Assessment Tool (EAT-10), ${ }^{10)}$ the Frequency Scale for the Symptoms of Gastroesophageal Reflux Disease (FSSG), ${ }^{11)} \mathrm{RSST},{ }^{12}$ ) the water swallowing test (WST), ${ }^{13)}$ and the simple swallow provocation test (SSPT) ${ }^{14)}$; tongue pressure measurement using a convenient disposable probe (JM-TPM; JMS, Hiroshima, Japan) ${ }^{15)}$; and skeletal muscle mass index measurement using the InBody 770 (Biospace, Seoul, Korea). These screening tests were carried out on the same day.

\section{Sensory Stimulation}

For IFC-TESS intervention, Gentle Stim (Careido, Kanagawa, Japan; Medical device certification number: 227AHBZX00026000) was used. It is a portable device that generates IFC with a beat frequency of $50 \mathrm{~Hz}$. After wiping the anterior cervical region with a wet towel (and shaving any excess body hair on this region), two pairs of electrodes were applied as specified in the Gentle Stim instructions. The intensity was set at the sensory threshold $(<3.0 \mathrm{~mA}$, typically around $2.0 \mathrm{~mA}$ ) where the subject felt a slight vibrating or tickling sensation. No muscle contraction was observed at this stimulation intensity. The stimulation was performed for 30 min per session. Two sessions were carried out each day, i.e., during the patients' lunch time and dinner time. This intervention was continued for 10 consecutive days. The first session was performed under the supervision of the respiratory physician, during which the nurse who was assigned to the patient's care on that day was instructed on how to use the device using a checklist. The checklist was posted on the patient's bedside. From the second session, nurses in the respiratory ward carried out the procedure, and physicians 


\section{Pre-intervention assessment}

1. COPD severity, history of exacerbation (from patient history and medical records)

2. Questionnaires on swallowing: EAT-10, FSSG

3. Swallow screening: RSST, WST, SSPT, tongue pressure, skeletal muscle mass index

\section{Intervention}

Who: Stable COPD patients, hospitalized for tests, patient education, rehabilitation etc.

What: IFC-TESS using the Gentle Stim ${ }^{\circledR}$ device (intensity: < 3.0mA)

When: During lunch and dinner, 30 minutes / session, 2 sessions / day for 10 consecutive days

Where: at the patient's bedside, in the respiratory medicine ward

How: The nurse in the respiratory medicine ward prepares and applies the device

\begin{tabular}{ll|}
\hline Post-intervention assessment \\
1. Questionnaires on swallowing: EAT-10, FSSG \\
2. Swallow screening: RSST, WST, SSPT, tongue pressure \\
3. Comparison of the parameters before and after intervention \\
\hline
\end{tabular}

Fig. 1. Study design. This was a three-phase study. First, the patient was assessed for swallowing function. Then, IFCTESS intervention was performed. After the 10-day intervention, the swallow screening was repeated.

were contacted whenever necessary.

After the 10-day intervention, the swallow screening tests (EAT-10, RSST, WST, and SSPT and tongue pressure measurement) were repeated. Then the test results before and after IFC-TESS intervention were compared.

\section{Statistical Analyses}

Descriptive statistics for baseline data are presented as medians and ranges. Differences between the data before and after IFC-TESS intervention were examined using McNemar's Chi-squared test or the Wilcoxon signed-rank test. A P-value of $<0.05$ was considered to indicate a statistically significant difference. All data analyses were conducted using JMP Pro software (ver. 15; SAS Institute, Cary, NC, USA).

\section{RESULTS}

Ten patients were included in the study, eight men and two women. The patient background data are shown in Table 1. The median age was 80 years (range, $71-86$ years), and the median body mass index was $17.3 \mathrm{~kg} / \mathrm{m}^{2}$ (range, $14.9-23.9 \mathrm{~kg} / \mathrm{m}^{2}$ ). In our patients, the severities of air-flow obstruction based on GOLD staging were as follows: I, 1; II, 1; III, 1; and IV, 7. The median number of exacerbations in
Table 1. Patient characteristics

\begin{tabular}{lc}
\hline & $\mathrm{n}=10$ \\
\hline Age, years & $80(71-86)$ \\
Male / female & $8 / 2$ \\
$\mathrm{BMI}, \mathrm{kg} / \mathrm{m}^{2}$ & $17.3(14.9-23.9)$ \\
Serum albumin, g/dl & $3.6(2.3-4.7)$ \\
$\mathrm{SMI}, \mathrm{kg} / \mathrm{m}^{2}$ & $4.9(4.0-7.6)$ \\
GOLD stage, I/II/III/IV & $1 / 1 / 1 / 7$ \\
Exacerbations in the past year & $2(0-5)$ \\
\hline
\end{tabular}

Data other than sex and GOLD stage are shown as median (range). BMI, body mass index; SMI, skeletal muscle mass index.

the previous year was 2 (range, $0-5$ ), and eight patients had experienced one or more exacerbations in the previous year. All patients had stable COPD and were independent in their daily lives in terms of both physical ability and cognitive status. There were no subjective symptoms of dysphagia.

The intervention was performed safely: there were no adverse events related to respiratory condition or skin irritation. There were two cases in which the physician was called because the stimulation did not attain the designated intensity. In both cases, reinforcing the tape fixation enabled the stimulation to work as intended. Otherwise, the nurses 

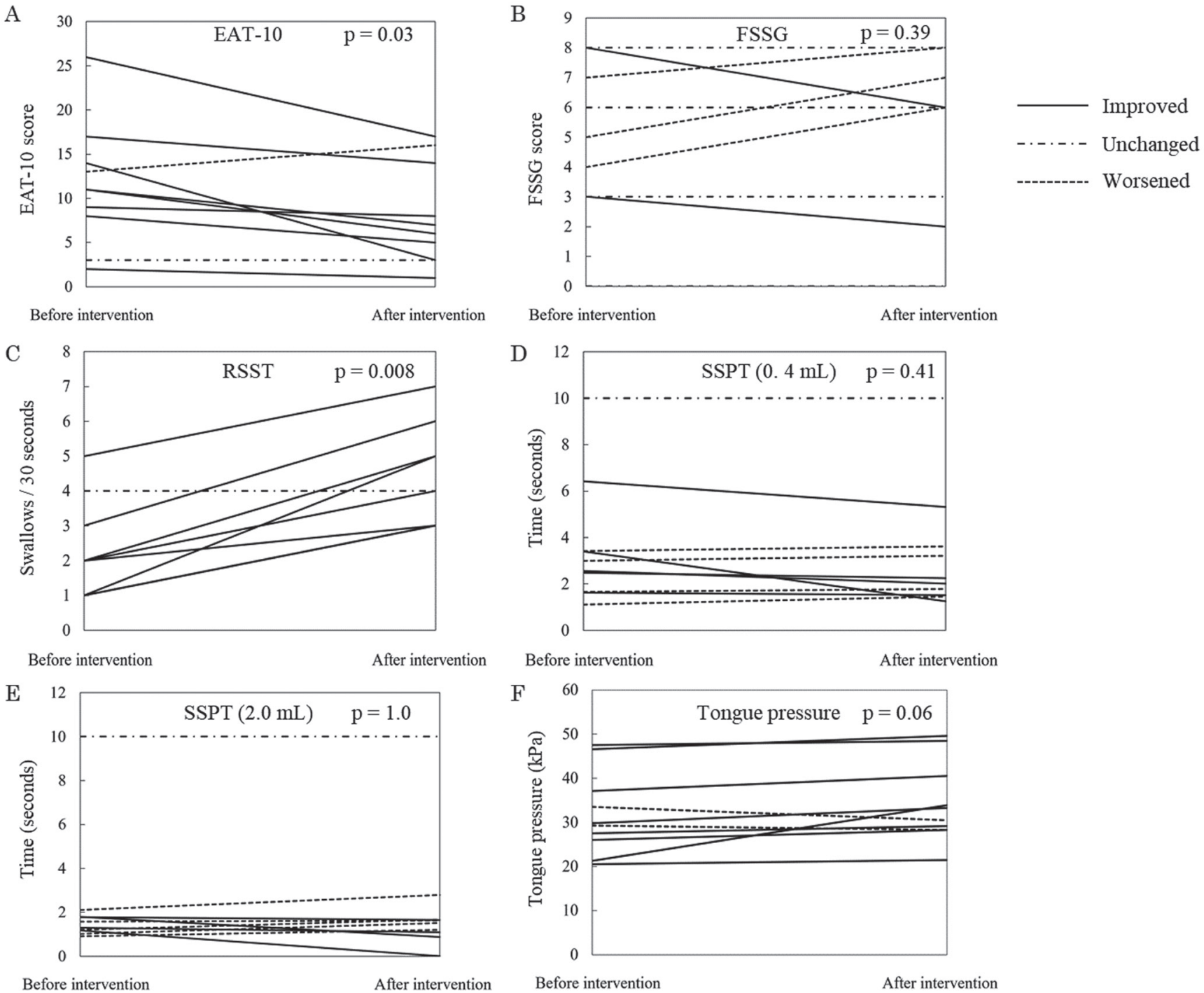

Fig. 2. Changes in each swallow screening test result before and after intervention. Statistical tests were performed using the Wilcoxon signed rank test.

were able to use the device without difficulty. No reports of discomfort, complaints, or concerns regarding the intervention were made by patients or nurses.

The swallow screening test results before and after IFCTESS intervention are shown in Fig. 2 and Table 2. The EAT-10 and RSST scores improved significantly after the intervention (Fig. 2A,C). The EAT-10 score decreased in eight patients, remained unchanged in one patient, and increased in one patient. There was a tendency for the subjective symptoms to improve; however, questions related to weight change and the ability to go out for meals remained unchanged. For RSST, the swallowing frequency increased in nine patients and did not change in one patient. There was no change in the number of patients who presented with abnormal results in the EAT-10, FSSG, and SSPT tests (Table 2). After the intervention, the number of patients showing abnormalities in RSST and EAT-10 tended to decrease, but the differences were not statistically significant (Table 2). Tongue pressure also showed a strong tendency to increase (Fig. 2F). The average of the three tongue pressure measurements increased in eight patients but decreased in the other two patients.

\section{DISCUSSION}

This was a prospective cohort study of the use of IFCTESS in stable hospitalized COPD patients. The results showed that IFC-TESS in the acute respiratory ward was safe and feasible. 
Table 2. Results of dysphagia screening before and after IFC-TESS intervention

\begin{tabular}{lcccc}
\hline Test & Criteria & Before intervention & After intervention & P value* \\
\hline EAT-10 & $\geq 3$ & 9 & 9 & NA \\
FSSG & $\geq 8$ & 2 & 2 & 1.0 \\
RSST & $\leq 5$ times/30 s & 10 & 8 & NA \\
\multirow{2}{*}{ WST } & (A) Vocal change & 6 & 3 & 0.25 \\
& (B) Drinking pattern** $2-5$ & 8 & 8 & NA \\
SSPT & (A) $0.4 \mathrm{ml}, \geq 3 \mathrm{~s}$ & 5 & 5 & 1.0 \\
& (B) $2.0 \mathrm{ml}, \geq 3 \mathrm{~s}$ & 1 & 1 & NA \\
\hline
\end{tabular}

Data are the number of patients who screened positive for each test.

NA, not applicable.

*McNemar's chi-squared test.

**Drinking patterns are defined as follows: 1, drinks $30 \mathrm{ml}$ in one swallow without choking; 2, drinks $30 \mathrm{ml}$ in multiple swallows without choking; 3, drinks $30 \mathrm{ml}$ in one swallow with some choking; 4, drinks $30 \mathrm{ml}$ in multiple swallows with some choking; 5, chokes and has difficulty drinking $30 \mathrm{ml}$.

IFC-TESS could be performed safely in patients with COPD. The contraindications for IFC-TESS do not include any aspects related to respiratory condition; nonetheless, there has been some speculation that IFC-TESS may entail risk in patients with an unstable respiratory condition. However, its safety was demonstrated in a previous retrospective study involving patients with acute aspiration pneumonia. ${ }^{16)}$ In comparison with a similar group of patients who did not undergo the intervention, the IFC-TESS group had no significant differences in their vital signs. Our results show additional prospective data for stable COPD patients, among which there were seven patients with stage IV COPD.

Regarding its feasibility, IFC-TESS could be performed daily without problems in a respiratory medicine ward of an acute tertiary hospital. We chose to apply the stimulation at mealtimes, during which pharyngeal movement is at its peak. The application of IFC-TESS during eating is expected to show similar effectiveness as applying it during swallow training with a speech therapist. No individual nurse was designated for the purpose of applying IFC-TESS; whichever nurse was responsible for the patient's care applied the electrodes, set the intensity, and remove the electrodes after the session. For the nurses, the time required for each session was no more than a few minutes in total, and it was manageable as a part of the daily care. The cost of the device plus the electrodes and tape for individual patients cannot be ignored. However, because the use of IFC-TESS is feasible without the intervention of specialists such as a speech therapist, we believe it to be a valuable option for patients and healthcare institutions. IFC-TESS also constitutes suitable and sustainable training for COPD patients who are often breathless, weak, and easily fatigued. Utilization of IFC-TESS in nurs- ing homes and home or outpatient care may also be a future topic of interest.

The effectiveness of IFC-TESS has been reported in patients with dysphagia ${ }^{7,8,17)}$ and aspiration pneumonia. ${ }^{16)}$ IFC-TESS also reportedly enhances saliva production in patients with dry mouth, ${ }^{18)}$ shortens pharyngeal latency in dysphagic patients, ${ }^{19)}$ and increases swallowing frequency in healthy men. ${ }^{17)}$ These changes are suspected to improve airway defense while also increasing oral intake. ${ }^{78}$ In the current study, the EAT-10 and RSST scores improved significantly after the intervention, as shown in Fig. 2. However, the number of patients who cleared the normal standard thresholds did not change significantly, as shown in Table 2. This is probably because the common threshold of the EAT10 and RSST are relatively strict. As shown in our previous study, the optimal cutoff value of swallow screening tests is different in stable COPD patients (in whom the swallowing disorder is often subclinical) than in more apparent swallowing disorders associated with stroke or neurological conditions. ${ }^{4,5)}$ Therefore, the significant differences shown in Fig. 2 may be of importance even if the results were unchanged with respect to the commonly applied thresholds (as in Table 2). Likewise, the lenient threshold of SSPT may be one of the reasons that the results of the SSPT were unchanged. There was also a strong tendency for the tongue pressure to improve by a small amount. The reported effects of IFC-TESS, such as enhanced saliva production, shortened pharyngeal latency, and increased swallowing frequency, all contribute toward the ability to perform more dry swallows in the RSST. Because EAT-10 is an indicator of the patients' subjective symptoms, it appears that these functional changes may also have a positive impact on the patients subjective 
swallowing issues. Moreover, it is possible that the increased swallowing frequency caused the tongue musculature to function more effectively, as reflected in the tendency for the tongue pressure to increase. However, there may be multiple factors, such as the patient becoming more familiar with the measurement technique and the effect of pulmonary rehabilitation being performed during hospitalization.

In two patients, the RSST increased to above the threshold of 5 times. When the RSST is 5 or less in patients with stable COPD, they are more prone to exacerbation in the following year. ${ }^{4)}$ In the same study, we showed that patients with an RSST of 6 or more had no moderate or severe exacerbations in the following year. Whether an improvement in the RSST decreases the risk of COPD exacerbation is a topic for further investigation.

In patients with $\mathrm{COPD}$, the unique changes in breathingswallowing coordination puts them at risk of aspiration. ${ }^{2)}$ Whereas swallowing usually occurs during exhalation, patients with COPD tend to inhale before or after the swallow, which risks residue and oral microbiota being aspirated. Aspiration should be prevented as much possible in these patients because their lung capacities are already compensated. Therefore, airway protection mechanisms are an important target for training in COPD patients. It is hoped that IFCTESS will be a feasible option to attain this, while also increasing oral intake and improving or preventing sarcopenia and other comorbidities of COPD.

There are several limitations to this study. First, it was a single center study with a small group of patients. The indicated feasibility and other results may differ depending on the setting. We believe that these data are of value as a pilot study. Second, there was no control group. Other factors such as medication, physiotherapy, or a placebo effect may have also affected the test results. However, there was no speech therapist involved in the study, and no specific swallow training was performed. Because the patients were in a stable state, the effect of other factors are believed to be minimal. Third, only the short-term effects of the IFC-TESS intervention are currently available. It is not clear whether the improvement in RSST will decrease the risk of future COPD exacerbation. However, it has been shown that a low RSST has a high impact on the risk of future exacerbation. ${ }^{4,5)}$ The long-term effects of IFC-TESS and improvements in RSST will be determined in the future.

This was the first study to investigate the utility of IFCTESS in stable COPD patients and also the first study to investigate its use during mealtimes. The 10-day intervention was safe, feasible, and significantly effective in improving
EAT-10 and RSST scores. The long-term effects of IFC-TESS await clarification in further studies with a statistically determined sample size and an adequate control group. Objective indicators, such as videoendoscopy and videofluoroscopy, and more precise indicators of oropharyngeal sensory function are also necessary. Additionally, the effectiveness of longer-duration sessions, more frequent sessions, a longer intervention period, or yearly interventions may also be of interest.

\section{CONCLUSION}

This study shows the safety, feasibility, and short-term effectiveness of IFC-TESS carried out during mealtimes in stable COPD patients. The long-term effects of IFC-TESS in these patients will be clarified in future studies.

\section{ACKNOWLEDGMENTS}

There was no funding associated with this study. The authors would like to express their deepest gratitude to the following members of the Iizuka Hospital Department of Respiratory Medicine for their contribution to this study: Yuri Hiramatsu, Takafumi Kawabata, Hiroaki Ohta, Mitsukuni Sakabe, Ryunosuke Ooi, Takuto Sueyasu, Saori Nishizawa, Kohei Yoshimine, Yuki Ko, Hiromi Ide, and Kosuke Tsuruno.

\section{CONFLICTS OF INTEREST}

The authors state that there are no conflicts of interest in connection with this article.

\section{REFERENCES}

1. Nagami S, Oku Y, Yagi N, Sato S, Uozumi R, Morita S, Yamagata Y, Kayashita J, Tanimura K, Sato A, Takahashi R, Muro S: Breathing-swallowing discoordination is associated with frequent exacerbations of COPD. BMJ Open Respir Res 2017;4:e000202. DOI:10.1136/bmjresp-2017-000202, PMID:28883930

2. Yoshimatsu Y, Tobino K, Nagami S, Yagi N, Oku Y: Breathing-swallowing discoordination and inefficiency of an airway protective mechanism puts patients at risk of COPD exacerbation. Int J Chron Obstruct Pulmon Dis 2020;15:1689-1696. DOI:10.2147/COPD. S257622, PMID:32764914 
3. Steidl E, Ribeiro CS, Gonçalves BF, Fernandes N, Antunes V, Mancopes R: Relationship between dysphagia and exacerbations in chronic obstructive pulmonary disease: a literature review. Int Arch Otorhinolaryngol 2015;19:74-79. PMID:25992155

4. Yoshimatsu Y, Tobino K, Sueyasu T, Nishizawa S, Ko Y, Yasuda M, Ide H, Tsuruno K, Miyajima H: Repetitive saliva swallowing test predicts COPD exacerbation. Int J Chron Obstruct Pulmon Dis 2019;14:2777-2785. DOI:10.2147/COPD.S226268, PMID:31824143

5. Yoshimatsu Y, Tobino K, Sueyasu T, Nishizawa S, Goto Y, Murakami K, Munechika M, Yoshimine K, Miyajima H: Repetitive saliva swallowing test and water swallowing test may identify a COPD phenotype at high risk of exacerbation. Clin Respir J 2019;13:321327. DOI:10.1111/crj.13014, PMID:30821113

6. Umezaki T, Sugiyama Y, Fuse S, Mukudai S, Hirano S: Supportive effect of interferential current stimulation on susceptibility of swallowing in guinea pigs. Exp Brain Res 2018;236:2661-2676. DOI:10.1007/s00221018-5325-0, PMID:29974148

7. Hara Y, Nakane A, Tohara H, Kubota K, Nakagawa K, Hara K, Yamaguchi K, Yoshimi K, Minakuchi $\mathrm{S}$ : Cervical interferential current transcutaneous electrical sensory stimulation for patients with dysphagia and dementia in nursing homes. Clin Interv Aging 2021;15:2431-2437. DOI:10.2147/CIA.S274968, PMID:33456308

8. Maeda K, Koga T, Akagi J: Interferential current sensory stimulation, through the neck skin, improves airway defense and oral nutrition intake in patients with dysphagia: a double-blind randomized controlled trial. Clin Interv Aging 2017;12:1879-1886. DOI:10.2147/ CIA.S140746, PMID:29158670

9. Vogelmeier CF, Criner GJ, Martinez FJ, Anzueto A, Barnes PJ, Bourbeau J, Celli BR, Chen R, Decramer M, Fabbri LM, Frith P, Halpin DM, López Varela MV, Nishimura M, Roche N, Rodriguez-Roisin R, Sin DD, Singh D, Stockley R, Vestbo J, Wedzicha JA, Agustí A: Global strategy for the diagnosis, management, and prevention of chronic obstructive lung disease 2017 report. GOLD executive summary. Am J Respir Crit Care Med 2017;195:557-582. DOI:10.1164/rccm.2017010218PP, PMID:28128970

10. Hidetaka W, Jun K: Translation, reliability, and validity of the Japanese version of the 10-item Eating Assessment Tool (EAT-10) for the screening of dysphagia. JJSPEN 2014;29:871-876. [in Japanese]
11. Kusano M, Shimoyama Y, Sugimoto S, Kawamura O, Maeda M, Minashi K, Kuribayashi S, Higuchi T, Zai H, Ino K, Horikoshi T, Sugiyama T, Toki M, Ohwada T, Mori M: Development and evaluation of FSSG: frequency scale for the symptoms of GERD. J Gastroenterol 2004;39:888-891. DOI:10.1007/s00535004-1417-7, PMID:15565409

12. Oguchi K, Saitoh E, Baba M, Kusudo S, Tanaka T, Onogi K: The repetitive saliva swallowing test (RSST) as a screening test of functional dysphagia (2). Validity of RSST. Jpn J Rehabil Med 2000;37:383-388. DOI:10.2490/jjrm1963.37.383

13. Kubota T, Mishima H, Hanada M, Namba I, Kojima Y: Dysphagia paralytica in cerebrovascular disease: screening test and its clinical application. Sogo Rihabiriteshon 1982;10:271-276. [in Japanese]

14. Terada K, Muro S, Ohara T, Kudo M, Ogawa E, Hoshino Y, Hirai T, Niimi A, Chin K, Mishima M: Abnormal swallowing reflex and COPD exacerbations. Chest 2010;137:326-332. DOI:10.1378/chest.09-0482, PMID:19783670

15. Hayashi R, Tsuga K, Hosokawa R, Yoshida M, Sato Y, Akagawa Y: A novel handy probe for tongue pressure measurement. Int J Prosthodont 2002;15:385-388. PMID:12170854

16. Nagami S, Maeda K, Fukunaga S, Ikeno M, Oku Y: Safety of transcutaneous electrical sensory stimulation of the neck in terms of vital parameters in dysphagia rehabilitation. Sci Rep 2019;9:13481. DOI:10.1038/ s41598-019-49954-9, PMID:31530879

17. Furuta T, Takemura M, Tsujita J, Oku Y: Interferential electric stimulation applied to the neck increases swallowing frequency. Dysphagia 2012;27:94-100. DOI:10.1007/s00455-011-9344-2, PMID:21607745

18. Hasegawa Y, Sugahara K, Sano S, Sakuramoto A, Kishimoto H, Oku Y: Enhanced salivary secretion by interferential current stimulation in patients with dry mouth: a pilot study. Oral Surg Oral Med Oral Pathol Oral Radiol 2016;121:481-489. DOI:10.1016/j. oooo.2016.01.017, PMID:27017403

19. Oku Y, Sugishita S, Imai T, Matsui T, Daimon T, Nozaki S, Yoshikawa H: Effects of short term interferential current stimulation on swallowing reflex in dysphagic patients. Int J Speech Lang Pathol Audiol 2015;3:1-8. DOI:10.12970/2311-1917.2015.03.01.1 